\section{A CASE OF CHOLECYSTITIS DUE TO THE TYPHOID BACILLUS.}

BY MARK W. RICHARDSON, M.D., BOSTON,

From the Pathological Laboratory of the Massachusetts General Hospital.

ABodt April 1, 1897, I had referred to me by Dr. Maurice H. Richardson two specimens for examination. The first consisted of about half a pint of dirty, brownish fluid, and the second was a swab soaked in that fluid for bacteriological investigation. The fluid had been evacuated from a gall-bladder which was the seat of a purulent inflammation, and which was distended by the impaction of a biliary calculus in the cystic duct.

I found the fluid to have the following characteristics: it was dirty-brown in color, cloudy and slightly viscid. The reaction was alkaline; the specific gravity 1.018 ; albumin one-half percent.; bile reaction very faint. There was much reddish-white, granular sediment. Microscopically, the sediment showed much normal blood (operation?) ; many small, round, fattydegenerated cells; considerable numbers of large, squamous epithelial cells; many bacilli in large clumps.

I next proceeded to the bacteriological examination of the swab above mentioned, sweeping it thoroughly over the surface of two blood-serum tubes. After twenty-four hours in the incubator each tube showed about half-a-dozen round, semi-transparent, whitishgray, moist colonies of considerable size. Microscopically the growth was found to be made up of what was apparently a pure culture of a short, stout bacillus with rounded ends. The organism varied considerably in size; occurred most often alone, but sometimes grew out into chains of from three to six elements; in short, the bacillus belonged apparently to the colon group, and was very probably the colon bacillus itself. I was much surprised, however, when, after further cultivation upon differential media, the organism showed all the characteristics of the typhoid bacillus: $(a)$ It was quite motile. (b) It produced no coagulation of litmus-milk, and only a very faint acid reaction. (c) It produced no gas when grown upon sugar-agar. (d) It showed no production of indol when grown in peptone solution. (e) It grew characteristically, without liquefaction, upon gelatin slant aud stab cultures. $(f)$ It showed no visible growth upon potato. $(g)$ It did not grow at all upon solution No. 1 of Proskauer and Capaldi. Upon solution No. 2 of the same authors it produced a marked acid reaction. (h) It showed the specific reaction of Pfeiffer when treated with typhoid serum.

The discovery of the typhoid organism in the purulent exudate added much to my interest in the case, and led me to inquire more carefully into the previous history. Dr. Chandler, of Townsend. Mass., the attending physician, gave me the following account of the case :

Five weeks previous to operation the patient, Mrs. $P$., an elderly lady, tirst came under observation with symptoms of an acute cystitis. This condition improved much after a week of appropriate treatment.

Four weeks before operation the temperature and pulse began to rise steadily. There was also nausea with considerable pain and tenderness in the right iliac region. The patient had a typhoidal look. The case was thought to be possibly one of appendicitis, and the patient was seen by Dr. M. H. Richardson, who advised waiting.

Two weeks before operation the temperature was still elevated. There was some pain in the right chest, and considerable cough with rusty sputum. No enlargement of the spleen was noted, and no rose-spots were seen. At this time a firm, rounded tumor was first made out below the ribs on the right side, and at about the level of the anterior superior spine of the ilium. The mass was very tender and painful. Examination under ether by Dr. Chaudler, of 'Townsend, and Dr. Stimson, of Fitchburg, showed that the tumor was quite movable, and apparently extended through to the back. A probable diagnosis of pyo- or hydronephrosis was made. Urine was said to be negative.

One week before operation the temperature had dropped to about $100^{\circ}$, but the tumor had become much larger, with considerable increase in pain aud teuderness.

At the time of operation no positive diagnosis was made, but the lesion was thought to be a pyo-nephrosis or an acute cholecystitis. A short incision in the back, bowever, showed the right kidney to be normal. An oblique cut was then made in front over the gall-bladder, which was found excessice.y distended. 'The gallbladder was first aspirated, then freely incised. Impacted in the cystic duct, and removed with great difficulty, was an elongated, cylindrical gall-stone with a smooth surface and rounded ends.

Since the operation the progress of the patient has been satisfactory. 'The opening in to the gall-bladder is gradually closing in, and there bas been great increase in weight and strength.

A week after the operation, at my request, Dr. Chandler sent me a specimen of the patient's blood (dried on paper), and a small portion of a stool. I could obtain no typhoid serum-reaction with the blood, however, nor did bacteriological cultivation from the stool by the Capaldi metbod show auy typhoid bacilli. In convection with this attempt it is interesting to note, nevertheless, that, although the dried blood caused no clumping of typhoid bacilli, the fluid from the gallbladder, when filtered and added, in proper proportion, to an independent typhoid culture, caused a wellmarked reaction. Indeed, if we look once more at the sediment of the inflammatory exudate, I think we must regard the large clumps of bacilli which we find there, as evidence of a gigantic serum-reaction which has taken place spontaneously in the gall-bladder.

From all the evidence obtainable in this case it seems clear that there was a typhoidal infection of the gallbladder associated with the presence of a gall-stone in the cystic duct. Was this infection primary or secondary?

A primary infection of the gall-bladder by the typhoid bacillus has, as far as I know, never been described. A priori, it is perfectly possible to imagine such a localized infection, but in the absence of postmortem evidence, such a supposition can have but little value.

On the other hand, a cholecystitis as a sequel to typhoid fever, as has been exhaustively shown by Dr. A. L. Mason ${ }^{1}$ is of quite frequent occurrence, and it is in this latter category that I would place the present case. For, as it seems to me, the history - four to five weeks of continued fever, abdominal pain and tenderness, typhoidal aspect, bronchitis - suggests 1 Boston Medical and Surgical Journal, May 13, 1897. 
distinctly a previous typhoidal invasion of the intestinal tract. Nor would the fact that the blood, one week after the beginning of convalescence, gave no typhoid reaction deter me from such a conclusion. For, as is well known, the blood-conditions necessary for the serum-reaction may be present upon one day and absent the next, or they may entirely disappear with or even before the fever, so that the value of a single negative result is not great.

As we look over the increasing literature upon the subject of cholecystitis as a sequel to typhoid fever, I tbink we cannot fail to be impressed with its great importance. Especially striking are the observations of Chiari, who found typhoid bacilli in the gall-bladders of 19 out of 22 typhoid cadavers. Moreover, out of these 19 positive cases 13 showed a condition of actual inflammation.

We see, therefore, that, in typhoid fever, the bile, far from being antagonistic to the growth of the typhoid bacillus, actually becomes a medium in which it reproduces itself rapidly. Furthermore, that the bacili may persist for considerable lengths of time in the gall-bladder is shown by the case of Gilbert and Girode, and that of Dupré (quoted by Mason, loc. cit.), in which cholecystotomy five and six months after typhoid fever showed pure cultures of the typhoid bacillus.

At the Massachusetts General Hospital during the last nine months ${ }^{2}$ cultures from the gall-bladder were made at three autopsies of typhoid patients, and in every case typhoid bacilli were demonstrated. Two of these cases were uncomplicated typhoids. The third was unusual in that it was combined with a general tuberculosis. This patient was admitted to the hospital November 2, 1896, and passed through what was apparently a typhoid fever lasting from three to four weeks. During this time a suspicious patch of consolidation was detected in one lung, but examinatiou of the sputum failed to reveal any tubercle bacilli. At the end of four weeks, when convalescence was apparently about to begin, irregular fever with cough, persistent vomiting and rapid emaciation set in, and death followed in six weeks. At the autopsy the following pathological conditions were found: chronic tuberculosis of the lungs, with cavity formation; chronic miliary tuberculosis of the liver, spleen and kidueys; chronic tubercular salpingitis, with localized abscess formation and circumscribed peritonitis; ulceration of the ileum, partly tubercular and partly typhoidal; tuberculosis of the mesenteric, retro-peritoneal, and bronchial lymph glands, etc. Typhoid bacilli were found in the bile, liver, kidney, and the pus of the pelvic abscess.

I could find no record as to the presence of a typhoid serum-reaction during the life of the patient, but a specimen of blood obtained at the autopsy, added to a typhoid culture, gave a well-marked positive result.

This case, therefore, not only illustrates the power of the typhoid bacillus to grow in the gall-bladder and to persist there for several weeks after the end of the proper typhoidal infection, but also demonstrates the possibility of a simultaneous double infection of the intestine with the bacilli of typhoid fever and tuherculosis. A very similar case has been reported recently by Guinon and Meunier. ${ }^{8}$

2 I am indebted to Drs. Fitz and Wright, of the Massachusetts Gen-

eral Hospital, for permission to publish these cases.
In the light of what has been said I think the possibility of a complicating cholecystitis should be borne strongly in mind in the later stages of any case of typhoid fever.

Given such an inflammation of the gall-bladder, and having failed to relieve the condition by medical methods, we should avoid the dangers of rupture or perforation by early resort to surgical interference.

RECORD OF TWO CASES OF BEGINNING PULMONARY TUBERCULOSIS TREATED WITH SUBCUTANEOUS INJECTIONS OF KOCH'S TUBERCULIN T. R. ${ }^{1}$

BY ELBRIDGE G. CUTLER, M.D., BOstoN.

Throvgr the courtesy of Mr. Humes Hall, the Boston representative of John T. Milliken \& Co., manufacturing chemists of St. Louis, Mo., I was enabled to give the injection of Koch's Tuberculin T. R. prepared by them in their laboratory to the following two cases of early pulmonary tuberculosis. The solutions were freshly prepared for each injection according to the printed directions. The injection was invariably given between or below the scapulæ. Each injection was given with an ordinary subcutaneous syringe after the first few trials of the antitoxin syringe of Williams. This necessitated filling the barrel of the syringe three times for each injection on an average, and the introduction of the needle into a new place each time.

There was no abscess produced in the eighty or more punctures which were made during the treatment of the cases. The only complaint was of a slight soreness in one case in the neighborhood of the injections on about the sixth day of treatment. The injections were also made in three other cases, but as these cases were not suitable to the treatment, from too advanced a stage of the tuberculous process, they have not been included in this report. No constitutional symptoms were observed after the injections in these two cases, nor did the temperature more than once rise as much as one degree Fahrenheit above its usual course. As a rule, the cough and local signs progressively diminished, the amount of the expectoration lessened, and the patient felt progressively better in all the cases in which I have used the Tuberculin 'T. R., though in the unfit cases (too advanced a stage of disease) emaciation and an extension of the disease kept steadily on.

Professor Koch states that only cases of beginning tuberculosis should be treated with Tuberculin 'I. R.; that the initial injection should not exceed one fivehundredth of a milligranue; that the temperature should be watched carefully ; and that no reaction fever of more than one-half a degree Centigrade should be allowed. If the fever due to the injection is more, the dose should be lessened; otherwise, every other day an injection may be given and the dose doubled each time till one has given fourteen injections or a final dose of sixteen milligrammes. It sometimes happens that more than the usual number of injections is required, or they may have to be made less often or smaller in amount each time. In order that a proper oversight of the patient may be insured and a careful record of the temperature taken it is necessary that the patient should be either treated in a hospital or have the ser1 Read before the Suffolk District Medical Society, October 30,1897. 